December 4, 2017

\title{
Theta vacuum in different gauges
}

\author{
C. Adam* \\ School of Mathematics, Trinity College, Dublin 2
}

\begin{abstract}
In some recent papers it is claimed that the physical significance of the vacuum angle theta for QCD-like theories depends on the chosen gauge condition. We criticise the arguments that were given in support of this claim, and show by explicit construction for the case of $\mathrm{QED}_{2}$ that and why they fail, confirming thereby the commonly accepted point of view.
\end{abstract}

*)email address: adam@maths.tcd.ie, adam@pap.univie.ac.at 


\section{Introduction}

In gauge theories where large gauge transformations (i.e. gauge transformations with a nonzero, integer winding number) exist, a new parameter, the vacuum angle $\theta$, enters the quantized theory, which is absent classically. The commonly accepted point of view is that this vacuum angle $\theta$ has physical consequences both for a pure Yang-Mills theory and for a QCD-like theory provided all the quarks are massive.

However, in some recent papers [1, 2, 3] (see also [4]) this point of view was criticised. There it was argued that the physical relevance or irrelevance of $\theta$ depends on the chosen quantization prescription. More precisely, it was claimed that whenever the Gauss law defining the space of physical states,

$$
\left.\left.\left.G^{\prime}[\lambda] \mid \text { phys }\right\rangle=0 \quad \text { or } \quad e^{i G[\lambda]} \mid \text { phys }\right\rangle \sim \mid \text { phys }\right\rangle
$$

(here $\lambda$ is a gauge function, and $G$ and $G^{\prime}$ are related), implements invariance w.r.t. small gauge transformations only, physics does not depend on $\theta$. In the opposite case, when gauge invariance w.r.t. both small and large gauge transformations is implemented via (1), physics depends on $\theta$.

As a consequence, in all covariant quantization prescriptions (like e.g. BRST quantization), where only "small" gauge invariance is implemented, physics should be independent of $\theta$. E.g., there would be no strong $\mathrm{CP}$ violation for $\theta \neq 0$, and the topological susceptibility would be zero.

In this paper first we briefly review and criticise the arguments that were presented by the authors of [1, 2, 3] in favour of their point of view. Both their arguments are quite general, so they should apply, e.g., to $\mathrm{QED}_{2}$ as well as to $\mathrm{QCD}_{4}$.

Therefore, we study the problem of the $\theta$ vacuum explicitly for pure two-dimensional electro-dynamics and for the massless and massive Schwinger models. We show that and why both arguments of [1, 2, 3] fail and confirm the commonly accepted point of view that physics depends on $\theta$ for pure electro-dynamics and for the massive Schwinger model, whereas it is independent of $\theta$ in the massless Schwinger model.

\section{Criticism of the arguments}

In [1, 2, 3] the following two arguments are given:

1. The topological charge density $\nu(x)$ is the divergence of a topological current $K_{\mu}$, $\nu=\partial_{\mu} K^{\mu}$. The topological charge operator $Q_{T}=\int d^{d-1} x K_{0}$ of this current relates different $\theta$ vacua,

$$
|\theta\rangle=e^{i \theta Q_{T}}|0\rangle
$$

as well as different Hamiltonians $H_{\theta}$ (with corresponding Lagrangians $L_{\theta}$ )

$$
H_{\theta}=e^{i \theta Q_{T}} H_{0} e^{-i \theta Q_{T}} \quad, \quad L_{\theta}=L_{0}+\int d^{d-1} x \theta \nu
$$


Therefore, the theory $H_{\theta},|\theta\rangle$ is unitarily equivalent to the theory $H_{0},|0\rangle$.

This argument fails when "large" gauge invariance is implemented in (1), because then the unitary operator $\exp \left(i \theta Q_{T}\right)$ does not commute with the Gauss operator $G[\lambda]$.

2. The VEV of the topological current is a constant in a covariant gauge,

$$
\left\langle\theta\left|K_{\mu}(x)\right| \theta\right\rangle=\left\langle\theta\left|K_{\mu}(0)\right| \theta\right\rangle=\text { const. }
$$

(where translation invariance of $|\theta\rangle$ is used) and, therefore, the topological density $\nu$ has zero VEV, $\partial_{\mu}\left\langle\theta\left|K^{\mu}(x)\right| \theta\right\rangle=\langle\theta|\nu(x)| \theta\rangle=0$. Further, the vacuum energy density $\epsilon$ is independent of $\theta$ because of $\langle\theta|\nu(x)| \theta\rangle \sim \frac{\partial \epsilon}{\partial \theta}$.

The current $K_{\mu}$ is gauge variant, but as the vacuum state $|\theta\rangle$ is a proper (normalized) state of the original, "large" Hilbert space (the one without a physical state condition that contains pure gauge excitations as well), the $\operatorname{VEV}\left\langle\theta\left|K_{\mu}\right| \theta\right\rangle$ nevertheless exists.

Our criticism of these two arguments is as follows.

ad 1) It is, of course, true that the two theories $H_{0},|0\rangle$ and $H_{\theta},|\theta\rangle$ are equivalent, but this is not the way the $\theta$ parameter enters the theory. Instead, it relies on the fact that, for a given theory $H_{0}$, the Gauss law (1) allows for a one-parameter family of states that may be parametrized by $\theta$. Therefore, the $\theta$-dependent theory is $H_{0}$, $|\theta\rangle$, which may be written equivalently as $H_{\theta},|0\rangle$ due to $(2),(3)$.

ad 2) The assumption on the existence of $\left\langle\theta\left|K_{\mu}\right| \theta\right\rangle$ is incorrect. A $|\theta\rangle$ state can only be constructed after the Gauss law (1) is implemented. This may be done e.g. by solving the Gauss law (see Section 3) or by explicitly constructing $|\theta\rangle$ states out of the gauge invariant (i.e. Gauss-law obeying) subspace of the conventional, perturbative Fock space (see Section 4). In both cases the Gauss law has to be implemented before a $|\theta\rangle$ state can be constructed. Therefore, the $\operatorname{VEV}\left\langle\theta\left|K_{\mu}\right| \theta\right\rangle$ does not exist.

In the following two sections we shall demonstrate our arguments by explicit construction, first in pure two-dimensional electro-dynamics, then in the Schwinger model.

\section{Pure electro-dynamics}

We use the conventions $\eta_{00}=-\eta_{11}=1, \epsilon_{01}=1$. The Lagrangian density for twodimensional electro-dynamics with a $\theta$ term reads $\left(F_{01} \equiv F\right)$

$$
\begin{gathered}
\mathcal{L}=-\frac{1}{4} F_{\mu \nu} F^{\mu \nu}-\frac{e \theta}{4 \pi} \epsilon_{\mu \nu} F^{\mu \nu}=\frac{1}{2} F^{2}+\frac{e \theta}{2 \pi} F \\
F_{\mu \nu}=\partial_{\mu} A_{\nu}-\partial_{\nu} A_{\mu} .
\end{gathered}
$$


First of all, as $\mathcal{L}$ only depends on $F$, we may, in principle, treat $F$ as the canonical field. Then we find the equation of motion (for the "Heisenberg field" $F$ )

$$
F=-\frac{e \theta}{2 \pi}
$$

and the Hamiltonian density

$$
\mathcal{H}=-\mathcal{L} .
$$

As the field $F$ is equal to a (c-number) constant, the theory is very trivial. There is only one physical state, the vacuum $|0\rangle$ (more precisely, $F$ does not generate new states out of the vacuum) and $F$ and $\mathcal{H}$ act on $|0\rangle$ like

$$
F|0\rangle=-\frac{e \theta}{2 \pi}|0\rangle \quad, \quad \mathcal{H}|0\rangle=\frac{1}{2}\left(\frac{e \theta}{2 \pi}\right)^{2}|0\rangle .
$$

Next, let us reanalyse the theory (5) when treating $A_{\mu}$ as the canonical field. In this case the equation of motion reads

$$
\partial_{\mu} F=0,
$$

i.e., again, $F$ is an (unspecified) constant.

Further, we want to define the topological density $\nu$, current $K_{\mu}$ and charge $Q_{T}$,

$$
\begin{gathered}
\nu=\frac{e}{4 \pi} \epsilon_{\mu \nu} F^{\mu \nu}=-\frac{e}{2 \pi} F_{01} \\
K_{\mu}=\frac{e}{2 \pi} \epsilon_{\mu \nu} A^{\nu} \\
Q_{T}=-\frac{e}{2 \pi} \int d x A_{1}(x)
\end{gathered}
$$

and the unitary operator

$$
U(\alpha)=e^{i \alpha Q_{T}}
$$

$\left(x \equiv x^{1}\right)$. We shall discuss the theory within canonical quantization and use the gauge condition $A_{0}=0$. Further, we rename the $\theta$ parameter in $\mathcal{L}$ into $\theta^{\prime}$. Then the Lagrangian density, canonical momentum $\Pi$ of the field $A_{1}$ and Hamiltonian density read

$$
\begin{gathered}
\mathcal{L}_{\theta^{\prime}}=\frac{1}{2}\left(\partial_{0} A_{1}\right)^{2}+\frac{e \theta^{\prime}}{2 \pi} \partial_{0} A_{1} \\
\Pi=-\partial_{0} A_{1}-\frac{e \theta^{\prime}}{2 \pi} \\
\mathcal{H}_{\theta^{\prime}}=\frac{1}{2}\left(\Pi+\frac{e \theta^{\prime}}{2 \pi}\right)^{2} .
\end{gathered}
$$

In $A_{0}=0$ gauge there remains the residual gauge freedom of time-independent gauge transformations $\lambda(x), A_{1}(x) \rightarrow A_{1}(x)+\frac{1}{e} \lambda^{\prime}(x)$, which is implemented by the Gauss operator $G[\lambda]\left(\lambda^{\prime} \equiv \partial_{1} \lambda\right)$,

$$
G[\lambda]=\frac{1}{e} \int d x \lambda^{\prime}(x) \Pi(x)
$$




$$
e^{i G[\lambda]} A_{1}(x) e^{-i G[\lambda]}=A_{1}(x)+\lambda^{\prime}(x) .
$$

Physical states should respect this symmetry, i.e., they should be invariant under $\exp (i G[\lambda])$, up to a c-number phase [5, 6],

$$
\left.\left.e^{i G[\lambda]} \mid \text { phys }\right\rangle=e^{i \rho[\lambda]} \mid \text { phys }\right\rangle,
$$

where $\rho$ is a real number that may depend on $\lambda$. In fact, any state on which the equation of motion (10) holds, obeys this Gauss law constraint. Let us define

$$
\Pi|\theta\rangle=-\frac{e \theta}{2 \pi}|\theta\rangle
$$

then we get

$$
e^{i G[\lambda]}|\theta\rangle=e^{-i \frac{\theta}{2 \pi} w[\lambda]}|\theta\rangle
$$

where

$$
w[\lambda]=\lambda(\infty)-\lambda(-\infty)
$$

and each $|\theta\rangle$ is a physical state. Obviously, the Gauss law (20) implies the more common local version of the Gauss law

$$
\left.\partial_{1} \Pi \mid \text { phys }\right\rangle=0,
$$

and both versions are equivalent on eigenstates of $\Pi,(21)$. The eigenvalue of the Hamiltonian density (17) is

$$
\mathcal{H}_{\theta^{\prime}}|\theta\rangle=\frac{1}{2}\left(\frac{e\left(\theta^{\prime}-\theta\right)}{2 \pi}\right)^{2}|\theta\rangle,
$$

therefore, here the combination $\left(\theta^{\prime}-\theta\right)$ is equivalent to $\theta$ in (9) (we could have chosen $\theta^{\prime}=0$ to make the equivalence even more obvious). Clearly, different $|\theta\rangle$ states are orthogonal. As $\exp (i G[\lambda])$ commutes with all the gauge invariant observables $\left(\Pi, \mathcal{H}_{\theta^{\prime}}\right.$ ), we may say that $\exp (i G[\lambda])$ defines a superselection rule, where different $|\theta\rangle$ states correspond to different sectors of the theory. Each sector contains precisely one state $|\theta\rangle$, the vacuum state in the given $\theta$ sector.

Observe that nothing so far depended on the boundary conditions of the gauge transformations $\lambda$, i.e., whether $w[\lambda]$ in (23) is arbitrary, $w[\lambda]=2 \pi m, m \in \mathcal{Z}$ (large gauge transformations), or $w[\lambda]=0$ (small gauge transformations). These restrictions just affect the value of the phase in $(22)$, i.e. $\exp (i G[\lambda])$ has the same eigenvalue 1 for all $\theta$ if $w[\lambda]=0$, and $\exp (i G[\lambda])$ has the same eigenvalue on $|\theta\rangle$ and on $|\theta+2 \pi\rangle$ if $w[\lambda]=2 \pi$. Nevertheless, $\Pi$ and $\mathcal{H}_{\theta^{\prime}}$ distinguish different $|\theta\rangle$ states, independently of these boundary conditions. In addition, $\Pi$ and $\mathcal{H}_{\theta^{\prime}}$ are invariant under gauge transformations $\lambda$ with arbitrary $w[\lambda]$, therefore there is no good reason for a restriction on $\lambda$.

The operator $U,(14)$, maps different $|\theta\rangle$ states into each other,

$$
\left|\theta+\theta^{\prime}\right\rangle=U\left(\theta^{\prime}\right)|\theta\rangle
$$

therefore we may use the operator $U$ to shift the $\theta$ dependence entirely to the Hamiltonian density via

$$
\mathcal{H}_{\theta^{\prime}-\theta}=U^{\dagger}(\theta) \mathcal{H}_{\theta^{\prime}} U(\theta)=\frac{1}{2}\left(\Pi+\frac{e\left(\theta^{\prime}-\theta\right)}{2 \pi}\right)^{2}
$$




$$
\mathcal{H}_{\theta^{\prime}-\theta}|0\rangle=U^{\dagger}(\theta) \mathcal{H}_{\theta^{\prime}}|\theta\rangle=\frac{1}{2}\left(\frac{e\left(\theta^{\prime}-\theta\right)}{2 \pi}\right)^{2}|0\rangle .
$$

Of course, it holds that

$$
\mathcal{H}_{\theta}|\theta\rangle=U(\theta) \mathcal{H}_{0} U^{\dagger}(\theta) U(\theta)|0\rangle=0
$$

but this does not imply the physical equivalence of different $|\theta\rangle$ states.

Next, let us comment on argument 2) concerning the VEV of the topological current $K_{\mu}$. In our case $K_{\mu} \sim \epsilon_{\mu \nu} A^{\nu}$, i.e. after gauge fixing $A_{0}=0$ we should study the VEV of $A_{1}$. For the introduction of the $|\theta\rangle$ states gauge invariance was essential, i.e. all $|\theta\rangle$ states obey the physical state condition (20). However, this physical state condition is incompatible with the existence of the $\operatorname{VEV}\left\langle\theta\left|A_{1}\right| \theta\right\rangle$, and, therefore, such a VEV does not exist.

So we have found for our theory that "physics" depends on $\theta$ and that different values of $\theta$ lead to different vacuum energy densities for all $\theta \in \mathcal{R}$. This conforms with the physical interpretation of $\theta$ as it was discussed in [7], where $\theta$ is interpreted as a background static electrical field $E=\frac{e \theta}{2 \pi}$. As nothing can screen this field (there is no matter), it may acquire arbitrary values.

Before we end this section, we want to show how a $2 \pi$ periodicity in $\theta$ may enter the theory, getting thereby also a clearer understanding of the role of the unitary operator $\mathrm{U},(14)$. Usually, the $2 \pi$ periodicity of $\theta$ is a consequence of the fact that only gauge fields with integer instanton numbers contribute to VEVs of physical observables. In our case obviously nothing restricts $F=\partial_{0} A_{1}$ (we choose $A_{0}=0$ gauge again) to integer instanton numbers (in fact, in the Euclidean version of the theory $F$ may be interpreted as a magnetic field perpendicular to the Euclidean plane, and this magnetic field may have arbitrary magnetic flux, which is proportional to the "instanton number" of $F$, see [8]). However, we may introduce this restriction as a constraint. This is done most conveniently in the path integral formalism,

$$
\begin{gathered}
Z=\sum_{n=-\infty}^{\infty} \int D F \delta\left(\int d^{2} x \frac{F}{2 \pi}-e n\right) e^{i \int d^{2} x\left(\frac{1}{2} F^{2}+\frac{e \theta}{2 \pi} F\right)} \\
=\sum_{n=-\infty}^{\infty} \int D F e^{i \int d^{2} x\left(\frac{1}{2} F^{2}+\left(\frac{\theta}{2 \pi}+n\right) e F\right)}
\end{gathered}
$$

and in the second line the $2 \pi$ periodicity of $\theta$ is obvious. So we have a whole ensemble of systems, numerated by $n$, and the Lagrangian $\mathcal{L}_{n}$ of system $n$ has an effective $\theta$ parameter $\theta^{\prime}=\theta+2 \pi n$. The corresponding Hamiltonian density is $\mathcal{H}_{n}=\frac{1}{2}\left(\Pi+\frac{e \theta}{2 \pi}+e n\right)^{2}$, see (17), provided that all the $\theta$ dependence is shifted to $\mathcal{H}_{n}$, i.e., the vacuum is $|0\rangle$.

The vacuum energy density of the whole ensemble of $\mathcal{H}_{n}$ is (remember that $\Pi|0\rangle=0$; $V$... space-time volume)

$$
\epsilon=-\frac{1}{V} \ln \left(\sum_{n} e^{-\frac{e^{2} V}{2}\left(\frac{\theta}{2 \pi}+n\right)^{2}}\right) .
$$


In the thermodynamic limit $V \rightarrow \infty$ in the sum within the logarithm only one term contributes, namely the one with $\left|\frac{\theta}{2 \pi}+n\right|=\min$. As $\theta$ varies, the only contributing $\mathcal{H}_{n}$ changes whenever a point $|\theta|=\pi+2 \pi m, m \in \mathcal{Z}$, is passed. This makes the vacuum energy density $2 \pi$ periodic in $\theta$ and equal to

$$
\epsilon=\frac{1}{2}\left(\frac{e(\theta+2 \pi n)}{2 \pi}\right)^{2} \quad \text { for } \quad-\pi-2 \pi n<\theta<\pi-2 \pi n
$$

and the contribution solely stems from $\mathcal{H}_{n}$ (similar arguments can be found in [9], [10]).

Here an important point is that on the whole ensemble $\sum_{n} \exp \left(-\int d x \mathcal{H}_{n}\right)$, the operator $U(2 \pi)$ acts as a symmetry, although an individual $\mathcal{H}_{n}$ is not symmetric under the action of $U(2 \pi)$. As $U(2 \pi)$ maps $U(2 \pi)|\theta\rangle=|\theta+2 \pi\rangle$, this is another way of stating the $2 \pi$ periodicity in $\theta$.

Observe that the whole discussion had nothing to do with the boundary conditions of gauge transformations. Still, each $\mathcal{H}_{n}$ in the ensemble is invariant under gauge transformations with arbitrary "winding number" $w[\lambda] / 2 \pi$, and there is no need to restrict, e.g., to integer winding number.

\section{Massless and massive $\mathrm{QED}_{2}$}

In the following we choose Lorentz gauge $\partial_{\mu} A^{\mu}=0$ (which is covariant), because there the wellknown operator solution [12, 13, 14] is at hand. In fact, our presentation will closely follow Chapter 10 of [13]. We use the $\gamma$-matrix conventions

$$
\gamma^{0}=\left(\begin{array}{cc}
0 & 1 \\
1 & 0
\end{array}\right), \quad \gamma^{1}=\left(\begin{array}{cc}
0 & -1 \\
1 & 0
\end{array}\right), \quad \gamma_{5}=\left(\begin{array}{cc}
1 & 0 \\
0 & -1
\end{array}\right)
$$

and by $c$ we always denote an unspecified constant whose precise value we do not need. The Lagrangian density of one-flavour $\mathrm{QED}_{2}$ is

$$
\mathcal{L}=\bar{\Psi}(i \not \partial+e A) \Psi-\frac{1}{4} F_{\mu \nu} F^{\mu \nu}-m \bar{\Psi} \Psi
$$

but we want to consider the massless case $m=0$ first. The quantum equations of motion are (in the following we bosonize the theory, and normal ordering w.r.t. the bosonic fields is always understood)

$$
\begin{gathered}
i \not \partial \Psi+e A \Psi=0 \\
\partial_{\mu} F^{\mu \nu}+e J^{\nu}=0 \\
\partial_{\mu} J_{5}^{\mu}=\frac{e}{2 \pi} \epsilon_{\mu \nu} F^{\mu \nu},
\end{gathered}
$$

where $(37)$ is the anomaly equation $\left(J_{5}^{\mu}=\epsilon^{\mu \nu} J_{\nu}\right)$. In Lorentz gauge $A_{\mu}$ may be written as

$$
A_{\mu}=-\frac{\sqrt{\pi}}{e}\left(\epsilon_{\mu \nu} \partial^{\nu} \Sigma+\partial_{\mu} \widetilde{\eta}\right)
$$




$$
F_{\mu \nu}=\frac{\sqrt{\pi}}{e} \epsilon_{\mu \nu} \square \Sigma,
$$

where $\widetilde{\eta}$ is a massless, free scalar field $(\square \widetilde{\eta}=0)$ that takes into account the residual gauge freedom (remember that each free, massless scalar field $\eta$ has a dual $\widetilde{\eta}$ via $\partial_{\mu} \eta=\epsilon_{\mu \nu} \partial^{\nu} \widetilde{\eta}$ ).

The Dirac equation is solved by

$$
\Psi=c e^{i \sqrt{\pi} \gamma_{5}(\Sigma+\eta)} \psi^{\mathrm{f}}
$$

where $\psi^{\mathrm{f}}$ solves the free Dirac equation and reads

$$
\psi^{\mathrm{f}}=c e^{i \sqrt{\pi}\left(\widetilde{\phi}+\gamma_{5} \phi\right)} \psi_{0}
$$

Here $\psi_{0}$ is the constant unit spinor, $\psi_{0}^{\mathrm{t}}=(1,1), \phi$ is another free, massless field and $\widetilde{\phi}$ is its dual.

From the Maxwell and anomaly equations we find $\square\left(\square+e^{2} / \pi\right) \Sigma=0$, i.e., $\Sigma$ is the sum of a free massless and a free massive (with Schwinger mass $e / \sqrt{\pi}$ ) field. Choosing $\Sigma$ to be purely a massive field, $\left(\square+e^{2} / \pi\right) \Sigma=0$ (which is possible), gives the following results: the vector current is

$$
J_{\mu}=-\frac{1}{\sqrt{\pi}} \epsilon_{\mu \nu} \partial^{\nu} \Sigma+L_{\mu} \quad, \quad L_{\mu}:=-\frac{1}{\sqrt{\pi}} \epsilon_{\mu \nu} \partial^{\nu}(\phi+\eta),
$$

where the free, massless field $\phi(41)$ is the prepotential of the free fermion current, $j_{\mu}^{\mathrm{f}}=$ $\bar{\psi}^{\mathrm{f}} \gamma_{\mu} \psi^{\mathrm{f}}=-(1 / \sqrt{\pi}) \epsilon_{\mu \nu} \partial^{\nu} \phi$. The Maxwell equation reads

$$
\epsilon_{\mu \nu} \partial^{\nu}\left(\square+\frac{e^{2}}{\pi}\right) \Sigma-\frac{e^{2}}{\sqrt{\pi}} L_{\mu}
$$

and cannot be fulfilled on the operator level. Instead, the space of physical states has to be defined via

$$
\left\langle\text { phys }\left|L_{\mu}\right| \text { phys }^{\prime}\right\rangle=0,
$$

and all operators $O$ leaving the space of physical states invariant, i.e. $\left\langle\right.$ phys $\left|L_{\mu} O\right|$ phys $\left.^{\prime}\right\rangle=$ 0 , may be chosen as observables. In addition, the theory may be equivalently represented by the bosonic Hamiltonian density

$$
\mathcal{H}_{\mathrm{bos}}=\frac{1}{2}\left(\left(\partial_{0} \Sigma\right)^{2}+\left(\partial_{1} \Sigma\right)^{2}+\frac{e^{2}}{\pi} \Sigma^{2}+\left(\partial_{0} \phi\right)^{2}+\left(\partial_{1} \phi\right)^{2}-\left(\partial_{0} \eta\right)^{2}-\left(\partial_{1} \eta\right)^{2}\right),
$$

where $\eta$ is quantized with opposite sign, $\left[\eta(t, x), \partial_{0} \eta(t, y)\right]=-i \delta(x-y)$, in order to maintain $\left\langle 0\left|\left[L_{\mu}(x), L_{\nu}(y)\right]\right| 0\right\rangle=0$, which follows from the physical state condition. Here $|0\rangle$ is the Fock vacuum of the fields $\Sigma, \eta$ and $\phi$ (which certainly is a physical state w.r.t. condition (44); for details see [13]).

Remark: shifting $\Sigma$ by a constant, $\Sigma \rightarrow \Sigma+c$, obviously is a symmetry of the underlying theory, see (38), (40), (41) (in fact, this shift just generates global chiral rotations $\Psi \rightarrow$ $\left.\exp \left(i \sqrt{\pi} \gamma_{5} c\right) \Psi\right)$. However, this manifest symmetry is lost in (45) due to the choice of 
$\Sigma$ as a purely massive field. Therefore, whenever we create a constant shift of $\Sigma$ by some symmetry transformation, we should compensate it by a redefinition of the $\Sigma$ field, $\Sigma^{\prime}=\Sigma+c$.

Now the construction of the $\theta$ vacuum proceeds as follows. The operator

$$
\chi=e^{2 \sqrt{\pi} i(\phi+\eta)}
$$

is an observable and commutes with the Hamiltonian density $\mathcal{H}_{\text {bos }}$. Therefore, it may be used to generate an infinite set of ground states out of the Fock vacuum $|0\rangle$,

$$
|n\rangle=\chi^{n}|0\rangle
$$

On physical states $\chi$ acts like a unitary implementer of large gauge transformations (with winding number one, see [13] and below), and we allow only for integer powers of $\chi$ in (47) (see below for a more detailed explanation of this point). The $|\theta\rangle$ vacuum is just the coherent superposition of these $n$-vacua, and $\chi$ is diagonal w.r.t. $|\theta\rangle$,

$$
|\theta\rangle=\sum_{n} e^{-i n \theta}|n\rangle \quad, \quad \chi|\theta\rangle=e^{i \theta}|\theta\rangle
$$

Now we should study the operators

$$
\begin{gathered}
U(\alpha)=e^{i \alpha Q_{T}}=e^{-i \frac{\alpha}{2 \sqrt{\pi}} \int d x\left(\partial_{0} \Sigma+\partial_{0} \eta\right)} \\
V(\alpha)=e^{-i \alpha Q_{5}}=e^{i \frac{\alpha}{2 \sqrt{\pi}} \int d x \partial_{0} \phi}
\end{gathered}
$$

where $Q_{5}$ is the chiral charge, $Q_{5}=1 / 2 \int d x j_{5,0}^{\mathrm{f}}$ (we choose the factor $1 / 2$ in order to have one unit of chiral charge for the chiral density $\left.\bar{\Psi}(1 / 2)\left(\mathbf{1}+\gamma_{5}\right) \Psi\right)$. Both operators associate the same charge $n$ to the $n$-vacuum $|n\rangle$,

$$
\begin{aligned}
& \left(\chi^{\dagger}\right)^{n} U(\alpha) \chi^{n}=e^{i n \alpha} U(\alpha) \\
& \left(\chi^{\dagger}\right)^{n} V(\alpha) \chi^{n}=e^{i n \alpha} V(\alpha),
\end{aligned}
$$

which demonstrates the fact that "instanton number equals chirality".

Both operators transform the $|\theta\rangle$ vacuum,

$$
U(\theta)\left|\theta^{\prime}\right\rangle=V(\theta)\left|\theta^{\prime}\right\rangle=\left|\theta+\theta^{\prime}\right\rangle .
$$

In addition, both operators leave the Hamiltonian $\mathcal{H}_{\text {bos }}$ invariant. For $V(\alpha)$ this is obvious, $V(\alpha) \mathcal{H}_{\text {bos }} V^{\dagger}(\alpha)=\mathcal{H}_{\text {bos. }} U(\alpha)$ shifts the field $\Sigma$ in $\mathcal{H}_{\text {bos }}$ to $\Sigma-\alpha$. However, as we remarked, this is due to a convention that hides the symmetry of the original theory under such a shift and should be compensated by a redefinition $\Sigma \rightarrow \Sigma-\alpha$.

Therefore, we conclude that the theory $H_{\theta},|0\rangle$ is equivalent to the theory $H_{0},|0\rangle$ in this case and, consequently, physics is independent of $\theta$ in the massless Schwinger model (here $|0\rangle$ denotes the $\theta$ vacuum for $\theta=0$, not the Fock vacuum). 
Now, let us briefly discuss the massive Schwinger model. The vacuum structure remains the same, but the bosonized Hamiltonian density (45) changes to

$$
\mathcal{H}_{m}=\mathcal{H}_{\text {bos }}+c m \cos (2 \sqrt{\pi}(\Sigma+\phi+\eta)) .
$$

Therefore, $\mathcal{H}_{m}$ is no longer invariant under the action of $V(\theta)$ or $U(\theta)$, but instead

$$
V(\theta) \mathcal{H}_{m} V^{\dagger}(\theta)=U(\theta) \mathcal{H}_{m} U^{\dagger}(\theta)=\mathcal{H}_{\text {bos }}+c m \cos (2 \sqrt{\pi}(\Sigma+\phi+\eta)+\theta)
$$

(where again a shift $\Sigma \rightarrow \Sigma-\theta$ has to be performed when the action of $U(\theta)$ is computed).

As a consequence, $H_{\theta},|0\rangle$ and $H_{0},|0\rangle$ are no longer equivalent and the $\theta$ dependence enters the massive Schwinger model in the wellknown fashion [15, 07, 13, 11, 16].

Concerning the existence of a VEV $\left\langle\theta\left|K_{\mu}\right| \theta\right\rangle$, we should observe that the vacuum raising operator $\chi,(46)$, acts as a constant operator only on gauge invariant operators. Therefore, a VEV of a gauge variant quantity like $K_{\mu}$ w.r.t. a $|n\rangle$ state is arbitrary and not translation invariant. Consequently, $K_{\mu}$ does not have a VEV w.r.t. the $|\theta\rangle$ vacuum.

We have presented our main arguments, but we want to add some comments before closing this section. In (44) the longitudinal vector $L_{\mu}$ vanishes only weakly, which has the advantage that the physical states just form a subset of the entire Fock space (of free fields for the massless Schwinger model; however, the combination $\eta+\phi$ remains a free, massless field even for nonzero fermion mass). As can be shown easily, $L_{\mu}$ generates zero norm states when applied to the Fock vacuum (or any other physical state). These zero norm states are redundant on gauge invariant observables, so one may define a new space of physical states by just dividing out the zero norm states. On this new space the physical state condition corresponding to (44) is

$$
\left.L_{\mu} \mid \text { phys }\right\rangle=0,
$$

which is similar to the local version (24) of the Gauss law in Section 3. At this point the question arises whether an integrated version of the Gauss law, analogous to (20), can be found, which could again give some insight into the role of winding gauge transformations. Actually, this may be done without problem. The Gauss operator $G[\lambda]$ that implements the residual gauge transformations on $\Psi$ and $A_{\mu}$,

$$
\begin{gathered}
e^{i G[\lambda]} \Psi(x) e^{-i G[\lambda]}=e^{i \lambda(x)} \Psi(x) \\
e^{i G[\lambda]} A_{\mu}(x) e^{-i G[\lambda]}=A_{\mu}(x)+\frac{1}{e} \partial_{\mu} \lambda(x),
\end{gathered}
$$

is given by

$$
G[\lambda]=\frac{1}{\sqrt{\pi}} \int d x^{1}\left((\eta(x)+\phi(x)) \partial_{1} \lambda(x)-(\widetilde{\eta}(x)+\widetilde{\phi}(x)) \partial_{0} \lambda(x)\right)
$$

as may be checked easily (see [13]). Remember that, because of the Lorentz gauge $\partial_{\mu} A^{\mu}=$ 0 , the residual gauge transformations $\lambda$ have to obey $\square \lambda=0$. Therefore, $\lambda$ may be decomposed like

$$
\lambda(x)=\lambda_{+}\left(x^{1}+x^{0}\right)+\lambda_{-}\left(x^{1}-x^{0}\right),
$$


and the Gauss operator may be rewritten, by using this decomposition and by some partial integrations, like

$$
\begin{gathered}
G[\lambda]=\int d x^{1}\left(-\left(\lambda_{+}+\lambda_{-}\right) L_{0}-\left(\lambda_{+}-\lambda_{-}\right) L_{1}\right)+ \\
\frac{1}{\sqrt{\pi}}\left((\eta+\phi)\left(w\left[\lambda_{+}\right]+w\left[\lambda_{-}\right]\right)-(\widetilde{\eta}+\widetilde{\phi})\left(w\left[\lambda_{+}\right]-w\left[\lambda_{-}\right]\right)\right)
\end{gathered}
$$

where

$$
w\left[\lambda_{ \pm}\right]=\lambda_{ \pm}(\infty)-\lambda_{ \pm}(-\infty),
$$

and we used the fact that $(\eta+\phi)($ and $(\widetilde{\eta}+\widetilde{\phi}))$ acts as a constant operator on all gauge invariant operators, and, consequently, on physical states. $L_{0}$ and $L_{1}$ are just the components of the longitudinal current $L_{\mu}$, see (42), (56). At this point we want to impose the restriction that the total bare electrical charge is zero,

$$
\int d x^{1} j_{0}^{\mathrm{f}}(x)=\int d x^{1} \partial_{1} \phi(x)=0
$$

This assumption is very reasonable because of the confining properties of the Schwinger model, and we already implicitly obeyed it by considering only uncharged operators as observables. (However, this assumption is by no means necessary; it is perfectly possible to include charged sectors and observables into the theory, which just somewhat complicates the vacuum structure, see [12, 13].) For the zero charge condition (63) to be respected by a gauge transformation (61), we have to assume that

$$
w\left[\lambda_{+}\right]-w\left[\lambda_{-}\right]=0 \quad \Rightarrow \quad w\left[\lambda_{+}\right]=w\left[\lambda_{-}\right]=\frac{1}{2} w[\lambda]
$$

In this case the Gauss operator simplifies to

$$
G[\lambda]=-\int d x^{1} \lambda^{\mu} L_{\mu}+\frac{1}{\sqrt{\pi}} w[\lambda](\eta+\phi),
$$

where we introduced the short-hand notation $\lambda^{0}:=\lambda_{+}+\lambda_{-}, \lambda^{1}:=\lambda_{+}-\lambda_{-}$.

Now it is very easy to write down the integrated version of the Gauss law analogous to $(20)$,

$$
\left.\left.e^{i G[\lambda]} \mid \text { phys }\right\rangle=e^{i \rho[\lambda]} \mid \text { phys }\right\rangle \text {. }
$$

Choosing

$$
e^{2 \sqrt{\pi} i(\eta+\phi)}|\theta\rangle \equiv \chi|\theta\rangle=e^{i \theta}|\theta\rangle
$$

as in (48), we find

$$
e^{i G[\lambda]}|\theta\rangle=e^{i \frac{\theta}{2 \pi} w[\lambda]}|\theta\rangle
$$

(see (22)), and, again, the integrated version, (66), of the Gauss law implies the local one, $(56)$. 
From the preceding discussion it is very clear where the $\theta$ dependence and $2 \pi$ periodicity come from: if we restrict the set of observables to operators with zero chirality (i.e. operators that do not contain $\chi$ ) we find no $\theta$ dependence (like for the Hamiltonian of the massless Schwinger model); if we restrict to operators with integer chiral charge (in our units $(50)$ ), i.e., operators containing integer powers of $\chi$ (like $\left.\bar{\Psi}(1 / 2)\left(\mathbf{1}+\gamma_{5}\right) \Psi=c \chi \exp (2 \sqrt{\pi} i \Sigma)\right)$, we find $2 \pi$ periodicity in $\theta$. Of course, this latter restriction is very reasonable from a physical point of view. The important point is that all these results have nothing to do with a possible restriction of the residual gauge transformations $\lambda$ (e.g. to integer or zero winding number).

What is affected by restrictions on $\lambda$ is the number of physical states that obey the integrated Gauss law (66). For unrestricted $\lambda$ the $|\theta\rangle$ states (67) are the only allowed states (of course, toghether with their excitations by the physical, massive $\Sigma$ field), and the Gauss law (66) defines a superselection rule. For integer winding number $w[\lambda]=2 \pi m$ linear combinations $\sum_{n} a_{n}|\theta+2 \pi n\rangle$ also obey the Gauss law (66), and for zero winding number $w[\lambda]=0$ arbitrary linear superpositions of $|\theta\rangle$ states obey the Gauss law (66), and the integrated and local versions of the Gauss law become equivalent. Such restrictions on $\lambda$, of course, cannot change the physical results we described so far. E.g., even if we allow for the Fock vacuum to be a physical state, it cannot be the vacuum state of the massive Schwinger model, because it is not even an eigenstate of the Hamiltonian $\mathcal{H}_{m}$. On the other hand, the $|\theta\rangle$ vacuum always belongs to the set of physical states, and VEVs of observables w.r.t. $|\theta\rangle$ do not depend on what restrictions are imposed on the residual gauge transformations $\lambda$. In addition, as already stated, there is no good reason to restrict $\lambda$, because all gauge invariant observables are invariant under gauge transformations $\exp (i G[\lambda])$ for arbitrary $\lambda$.

\section{Summary}

We have criticised the arguments of [1, 2, 3] by explicit construction of the simplest models where they should apply, and demonstrated their failure, which was the purpose of this article. We showed that the implementation of invariance for arbitrary, large or small gauge transformations via the Gauss law (1) just affects the number of states that fulfill the Gauss law. The $|\theta\rangle$ vacuum always obeys the Gauss law, and VEVs of observables w.r.t. $|\theta\rangle$ do, of course, not depend on the number of further states that are permitted by the Gauss law. We confirmed that the vacuum energy densities of pure two-dimensional electro-dynamics and of the massive Schwinger model depend on $\theta$, whereas the vacuum energy density of the massless Schwinger model is independent of $\theta$, as expected.

\section{Acknowledgement}

The author thanks the members of the Department of Mathematics at Trinity College, where this work was performed, for their hospitality. Further thanks are due to R. Jackiw for helpful comments. 


\section{References}

[1] H. Yamagishi, I. Zahed, hep-th 9709125

[2] H. Yamagishi, I. Zahed, hep-ph 9507296

[3] H. Yamagishi, Prog. Theor. Phys. 87 (1992) 785

[4] H. Kikuchi, Int. J. Mod. Phys. A9 (1994) 2741

[5] R. Jackiw, C. Rebbi, Phys. Rev. Lett. 37 (1976) 172

[6] C. Callan, R. Dashen, D. Gross, Phys. Lett. B63 (1976) 334

[7] S. Coleman, Ann. Phys. 101 (1976) 239

[8] M. P. Fry, Phys. Rev. D45 (1992) 682, D47 (1993) 2629

[9] A. V. Smilga, Phys. Rev. D54 (1996) 7757

[10] I. Halperin and A. Zhitnitsky, hep-ph 9711398

[11] C. Adam, Ann. Phys. 259 (1997) 1

[12] J. Lowenstein, J. Swieca, Ann. Phys. 68 (1971) 172

[13] E. Abdalla, M. Abdalla, K. D. Rothe, "2 dimensional Quantum Field Theory", World Scientific, Singapore 1991

[14] L. V. Belvedere, K. D. Rothe, B. Schroer, J. A. Swieca, Nucl. Phys. 153 (1979) 112

[15] S. Coleman, R. Jackiw, L. Susskind, Ann. Phys. 93 (1975) 267

[16] C. Adam, Phys. Lett. B 363 (1995) 79 